\title{
In vitro study of neural stem cells and activated Schwann cells cocultured on electrospinning polycaprolactone scaffolds
}

This article was published in the following Dove Press journal:

Journal of Neurorestoratology

6 September 2017

Number of times this article has been viewed

\author{
Baoyou Fan ${ }^{1,2, *}$ \\ Xianhu Zhou ${ }^{1,2, *}$ \\ Lina Wang ${ }^{3, *}$ \\ Zhijian Wei ${ }^{1,2}$ \\ Wei Lin ${ }^{1,2}$ \\ Yiming Ren ${ }^{1,2}$ \\ Guidong Shi ${ }^{1,2}$ \\ Xin Cheng ${ }^{1,2}$ \\ Lianyong Wang ${ }^{3}$ \\ Shiqing Feng ${ }^{1,2}$ \\ 'International Science and \\ Technology Cooperation Base of \\ Spinal Cord Injury, Department \\ of Orthopedic Surgery, Tianjin \\ Medical University General Hospital, \\ ${ }^{2}$ Tianjin Neurological Institute, Key \\ Laboratory of Post-neuroinjury \\ Neuro-repair and Regeneration in \\ Central Nervous System, Ministry \\ of Education and Tianjin City, ${ }^{3}$ Key \\ Laboratory of Bioactive Materials, \\ Ministry of Education, College of Life \\ Sciences, Nankai University, Tianjin, \\ People's Republic of China \\ *These authors contributed equally to \\ this work
}

Correspondence: Shiqing Feng International Science and Technology Cooperation Base of Spinal Cord Injury,

Department of Orthopedic Surgery,

Tianjin Medical University General

Hospital, 4 Anshan Road, Heping District,

Tianjin 300052, People's Republic of China

Tel +86 I3920286292

Email sqfeng@tum.edu.cn

Lianyong Wang

Key Laboratory of Bioactive Materials,

Ministry of Education, College of Life

Sciences, Nankai University, 94 Weijin

Road, Nankai District, Tianjin 30007I,

People's Republic of China

Tel +86 I39 20286292

Emailwly@nankai.edu.cn
Background: To investigate the biocompatibility of electrospinning polycaprolactone (PCL) fiber scaffolds and coculture system, which consisted of neural stem cells (NSCs) and activated Schwann cells (ASCs).

Materials and methods: ASCs were isolated from sciatic nerves, ligated for 7 days, in 4-week-old Wistar rats, and the NSCs were isolated from the hippocampus of E14.5 Wistar rat embryos. ASCs, NSCs and ASCs combined with NSCs were 3D cultured on the electrospinning PCL fiber scaffolds. Crystal violet staining was used to find the suitable density of ASCs for growth, and the proliferation of NSCs and ASCs were tested by Cell Counting Kit (CCK)-8 assay, and cell adhesion, differentiation of NSCs and myelin basic protein (MBP) expression of ASCs were observed by laser confocal microscopy. Distribution and morphology were assessed by scanning electron microscopy.

Results: The average diameter of fibers in electrospinning PCL scaffolds was approximately $7.93 \pm 1.41 \mu \mathrm{m}$. ASCs could grow well at the density of $2 \times 10^{4} / \mathrm{cm}^{2}$, and a certain number of cells extended along the longitudinal axis of fibers, and the shape of the cells was spindle, which was consistent with crystal violet staining results. The CCK-8 experiment showed ASCs could proliferate gradually on the PCL scaffold within 7 days, as well as NSCs, and NSCs differentiated into astrocytes, neurons and oligodendrocytes on the PCL scaffold; PCL scaffolds could improve the differentiation rate of neurons. After NSCs and ASCs were cocultured on electrospinning PCL scaffolds, ASCs could express MBP and NSCs could differentiate into neurons, which distributed around those ASCs expressing MBP.

Conclusion: Electrospinning PCL fibrous scaffolds showed good biocompatibility, and the fibers had an inducing effect on the distribution of ASCs. NSCs and ASCs cultured on electrospinning PCL scaffolds could form 3D culture system, and NSCs could differentiate into neurons which distributed around the ASCs expressing MBP.

Keywords: neural stem cells, Schwann cells, PCL, scaffold, electrospinning

\section{Introduction}

Nerve repair has long been a major issue in medicine which is difficult to overcome. The current researches focused on cell transplantation, a promising strategy for nervous system repair. ${ }^{1-4}$ The transplanted cells could replace the injured cells and remodel the native tissues. In recent years, a variety of kinds of cells have been used for the neural repair. ${ }^{2,5-8}$ However, single-cell transplantation strategy cannot achieve a satisfying repair effect. In recent studies, combined strategy is widely used.

Tissue engineering involves cells, factors and scaffolds. The cells commonly used in tissue engineering are neural stem cells (NSCs), Schwann cells, bone marrow mesenchymal stem cells, olfactory cells, etc. ${ }^{9} \mathrm{NSCs}$, with the ability to differentiate into 
neurons, astrocytes and oligodendrocytes, are the most promising candidate cells for neural repair. A considerable amount of studies demonstrated that NSCs could repair the damage of central or peripheral nervous system. ${ }^{10-14}$ However, transplantation of NSCs has shortcomings: the survival rate of NSCs is low; ${ }^{15}$ most NSCs differentiate into astrocytes; and cavities are observed to exist after NSCs transplantation. ${ }^{10,11,16,17}$

Another important factor in tissue engineering is growth factors. The main focus of research is on neurotrophic factors, which include brain-derived neurotrophic factor (BDNF), nerve growth factor (NGF) and neurotrophin-3 (NT-3). Generally, neurotrophic factors are added to the scaffold material; ${ }^{18-20}$ however, this method is not effective. After the peripheral nerve injury, Schwann cells transform into a nonmyelinated state, "activated state". ${ }^{21}$ Activated Schwann cells (ASCs) could be isolated from preinjured peripheral nerves. ${ }^{22}$ ASCs have the ability to secrete a variety of neurotrophic factors to promote the growth of axons, such as BDNF and NGF and the myelin formation of central and peripheral axons, which enhances the conduction of axons. They also express a variety of adhesion factors and some membrane receptors, and suppress the formation of scar and cavity. ${ }^{1,23}$ Thus, ASCs could be the source of neurotrophic factors to support NSCs.

After cell transplantation, most cells die without the support of substrate, and especially, the cavity exists after transplantation in spinal cord injury. ${ }^{24}$ The scaffolds composed of biodegradable and biocompatible materials can bridge the cavity and provide support for the survival of cells. ${ }^{25}$ Polycaprolactone (PCL) is nontoxic, biodegradable and has fair mechanical strength. PCL is decomposed through tricarboxylic acid cycle. PCL has been approved by the US FDA and used in many products, such as absorbable sutures, dressings, ${ }^{26,27}$ contraceptives and internal fixation. ${ }^{28}$ It has a longer degradation period, and therefore, it can be utilized as a carrier for cell transplantation to provide support for cells. In recent years, PCL was used to repair spinal cord injury and peripheral nerve injury in many studies, ${ }^{29-31}$ but few studies have investigated the biocompatibility of PCL and coculture system, that is, NSCs combined with ASCs.

In this study, we assessed the proliferation and distribution of ASCs and NSCs, and the neural differentiation of NSCs on PCL scaffolds. Finally, the biocompatibility of PCL scaffolds and coculture system was assessed.

\section{Materials and methods Isolation and culture of cells}

\section{Animals}

Four-week-old Wistar rats were purchased from the Laboratory Animal Center of the Academy of Military Medical Sci- ences (Beijing, People's Republic of China). All experiments were approved by the Ethics Committee of Tianjin Medical University and complied with the US National Institutes of Health Guide for the Care and Use of Experimental Animals.

\section{Neural stem cells}

The procedure of NSCs isolation referred to the methods of Azari $^{32}$ with modification. NSCs were isolated from the hippocampus part of the brain of ED 14.5 Wistar rat embryos. Papain was utilized to digest tissue pieces. The cells were plated at a density of $5 \times 10^{5} / \mathrm{mL}$ in complete NSCs medium, and $10 \mathrm{~mL}$ complete NSCs medium was used for T75 flasks. Complete NSCs medium included neurobasal medium (Gibco 21103-049), $20 \mathrm{ng} / \mathrm{mL}$ epidermal growth factor (Gibco PHG0311), $20 \mathrm{ng} / \mathrm{mL}$ basic fibroblast growth factor (Gibco PHG0026), 0.5\% penicillin-streptomycin (Gibco 15140163), 2\% B27 supplement (Gibco 17504-044) and 1\% Glutamax (Gibco 35050-061). The third-passage NSCs were used for further experiment.

\section{Activated Schwann cells}

ASCs were obtained from sciatic nerves, preinjured for 7 days by ligation, in 4-week-old Wistar rats. Rats were immersed in $75 \%$ ethanol for 10 min after sacrifice, and sciatic nerves were taken out and cut into pieces. Then, the pieces were digested in collagenase II for $2 \mathrm{~h}$. The digestion was stopped with complete culture media, and cells were cultured in T75 flasks. Complete medium contained DMEM-F12 (Gibco 11330032), 10\% fetal bovine serum (Corning-Cellgro R35$076-\mathrm{cv}$ ) and $1 \%$ penicillin-streptomycin.

\section{Fabrication of PCL scaffolds}

PCL fiber scaffolds were prepared by electrospinning. PCL was dissolved in a mixture of methanol and chloroform at a ratio of 1:5 (v/v) to obtain $15 \mathrm{wt} \%$ PCL spinning solution. The spinning solution was added to the spinning tube with a jet flow rate of $6 \mathrm{~mL} / \mathrm{h}$, and the electric field strength was $+17 \mathrm{kV}$ at normal room temperature and pressure. The PCL fibers were collected at $150 \mathrm{rpm}$ on a rotating shaft which was $17 \mathrm{~cm}$ from the discharge port. PCL scaffolds were provided by Key Laboratory of Bioactive Materials, Ministry of Education, College of Life Sciences, Nan Kai University.

\section{Cell seeding on scaffolds}

PCL scaffolds were made to suit 48-well and 96-well plates by a punch process done under ultraviolet light for $1 \mathrm{~h}$, and then they were laid in well with a circle to weight it down. In order to find the suitable density for culturing ASCs on 
PCL scaffolds, we examined different density of cells: $2 \times 10^{4} /$ $\mathrm{cm}^{2}, 8 \times 10^{3} / \mathrm{cm}^{2}$ and $6 \times 10^{3} / \mathrm{cm}^{2}$. After 3 days of culture on the scaffolds, crystal violet staining was performed. The samples were washed with PBS three times, and fixed in 4\% paraformaldehyde at room temperature for $10 \mathrm{~min}$. They were again washed with PBS three times, and then crystal violet solution was added. After $5 \mathrm{~min}$, the samples were observed under microscope.

For ASCs, single-cell suspension was prepared at a density of $4 \times 10^{5}$ cells $/ \mathrm{mL}$, and then $50 \mu \mathrm{L}$ cell suspension was added on scaffolds. For NSCs, cell suspension was prepared at a density of $5 \times 10^{5}$ cells $/ \mathrm{mL}$, and then $100 \mu \mathrm{L}$ cell suspension was added on scaffolds. Then, $500 \mu \mathrm{L}$ cell culture medium was added $2 \mathrm{~h}$ later. For coculture, ASCs were cultured on PCL scaffolds for 3 days, and then NSCs were added on scaffolds.

\section{Proliferation assessment}

CCK-8 (C0038; Beyotime Biotechnology, Shanghai, People's Republic of China) was used to assess the proliferation of cells on scaffolds on 1, 3, 5 and 7 days. For this, $2 \times 10^{4}$ ASCs and $3 \times 10^{4}$ NSCs were separately seeded on PCL membrane in 48-well and 96-well plates. At every time point, five parallel samples were measured. First, culture medium was aspirated, and the prepared CCK-8 solution was added to each well for $2 \mathrm{~h}$ at $37^{\circ} \mathrm{C}$. Then, the reaction solution was removed into a new 96-well plate, and OD of the solution was measured at $450 \mathrm{~nm}$.

\section{Immunofluorescence staining}

Cells cultured on cover glass were fixed in $4 \%$ paraformaldehyde at room temperature for $10 \mathrm{~min}$, washed with PBS three times and incubated in blocking solution (5\% serum $+0.25 \%$ Triton $\mathrm{X}-100$ ) for $1 \mathrm{~h}$ at room temperature. The cells were incubated with primary antibodies overnight at $4{ }^{\circ} \mathrm{C}$. Primary antibodies included S-100 (1:200), $\beta \mathrm{III}-$ tubulin (1:500; Abcam, Cambridge, UK), GFAP (1:1000; Abcam), O4 (1:400; R\&D Systems), Nestin (1:100; Abcam) and myelin basic protein (MBP) (1:200; Abcam). On the next day, the cells were washed with PBS three times, and incubated with appropriate secondary antibodies for $2 \mathrm{~h}$ at room temperature. Secondary antibodies included goat anti-rabbit (1:500; Beyotime Biotechnology), goat anti-rat (1:500; Santa Cruz), donkey anti-rabbit (1:500; Beyotime Biotechnology) and donkey anti-goat antibodies (1:500; ProteinTech, Wuhan, People's Republic of China). Then, cells were washed with PBS three times, and incubated with DAPI (1:100) for $5 \mathrm{~min}$. Finally, after washing three times with PBS, cover glasses were covered with slides. Fluorescence was detected by a fluorescence microscope (IX71; Olympus, Tokyo, Japan).

\section{Confocal micrographs}

The procedure of sample preparation was similar to immunofluorescence staining. The samples were observed with an Olympus FV300 microscope. Ten distinct images were acquired for one sample, and eleven photographs per field were obtained along the z-axis.

\section{Scanning electron microscopy}

PCL scaffolds or scaffolds with cells were fixed in $2.5 \%$ glutaraldehyde overnight at $4^{\circ} \mathrm{C}$. After washing with PBS three times, samples were dehydrated with a series of graded ethanol $(60 \%, 70 \%, 80 \%, 90 \%, 100 \%)$, and then freeze-dried for 2 days. The samples were covered with gold using sputter coating. The morphologies of cells and scaffolds were observed by using scanning electron microscopy (SEM, Zeiss Ultra 55; Carl Zeiss, Oberkochen, Germany) under an accelerating voltage of $15 \mathrm{kV}$. The images were handled with an image analysis software (ImageJ; National Institutes of Health, Bethesda, MD, USA). At least five images from three samples for each group were used for the quantification.

\section{Statistical analysis}

All quantitative data are reported as mean \pm standard deviation. Two groups were compared using analysis of variance (ANOVA), and analyses were performed through SPSS software (version 22.0). $P<0.05$ was considered to be statistically significant.

\section{Results \\ Structural characterization of PCL scaffolds}

PCL scaffolds used in the present study were prepared by electrospinning technique, and the membranes were designed in two different sizes by a punch process: 0.16 and $0.8 \mathrm{~cm}^{2}$. Fibers were arranged randomly. The diameter of 100 randomly selected fibers was counted through Image-Pro 6, and the results showed that mean diameter of these fibers in PCL scaffolds used in the present study was $7.93 \pm 1.41 \mu \mathrm{m}$ (Figure 1).

\section{Identification of NSCs and ASCs}

The identification of ASCs was examined by S-100/DAPI immunofluorescence staining at the third generation, and the results showed that the percentage of cells which could express S-100 in ASCs was $97.12 \pm 0.72 \%$. The morphology of most cells was spindle type (Figure 2A-C).

NSCs of the third generation were used to detect their identification and differentiation ability. As shown in 

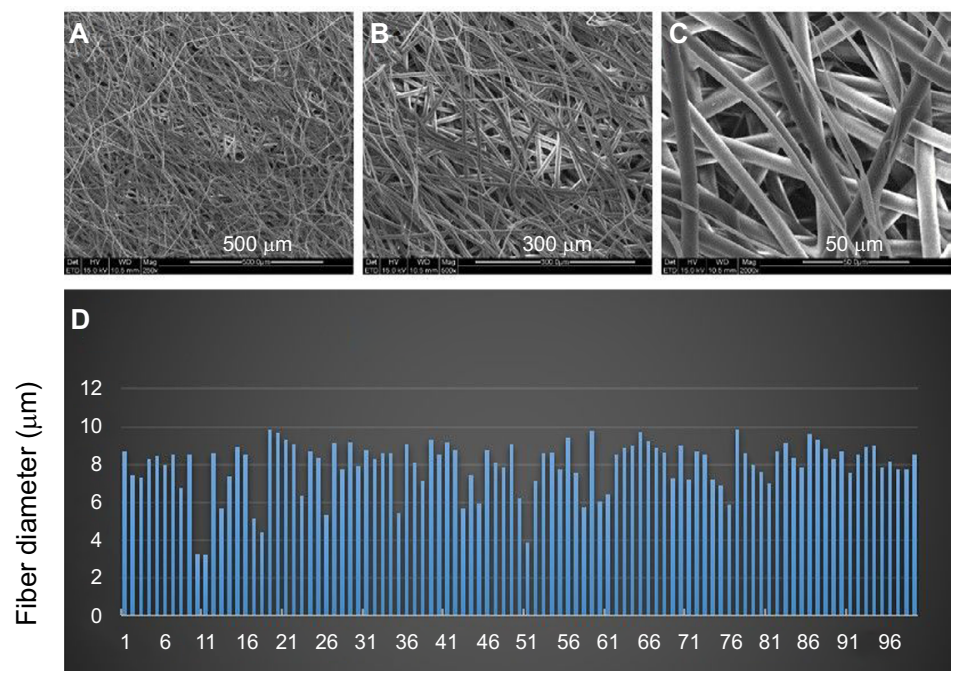

Number of fibers

Figure I Characteristics of PCL fiber scaffolds observed by SEM.

Notes: SEM images of PCL scaffold at different magnifications (A: $500 \mu \mathrm{m}$; B: $300 \mu \mathrm{m}$; and C: $50 \mu \mathrm{m}$ ). (D) Histogram of the diameters of 100 PCL fibers selected randomly. Abbreviations: PCL, polycaprolactone; SEM, scanning electron microscopy.
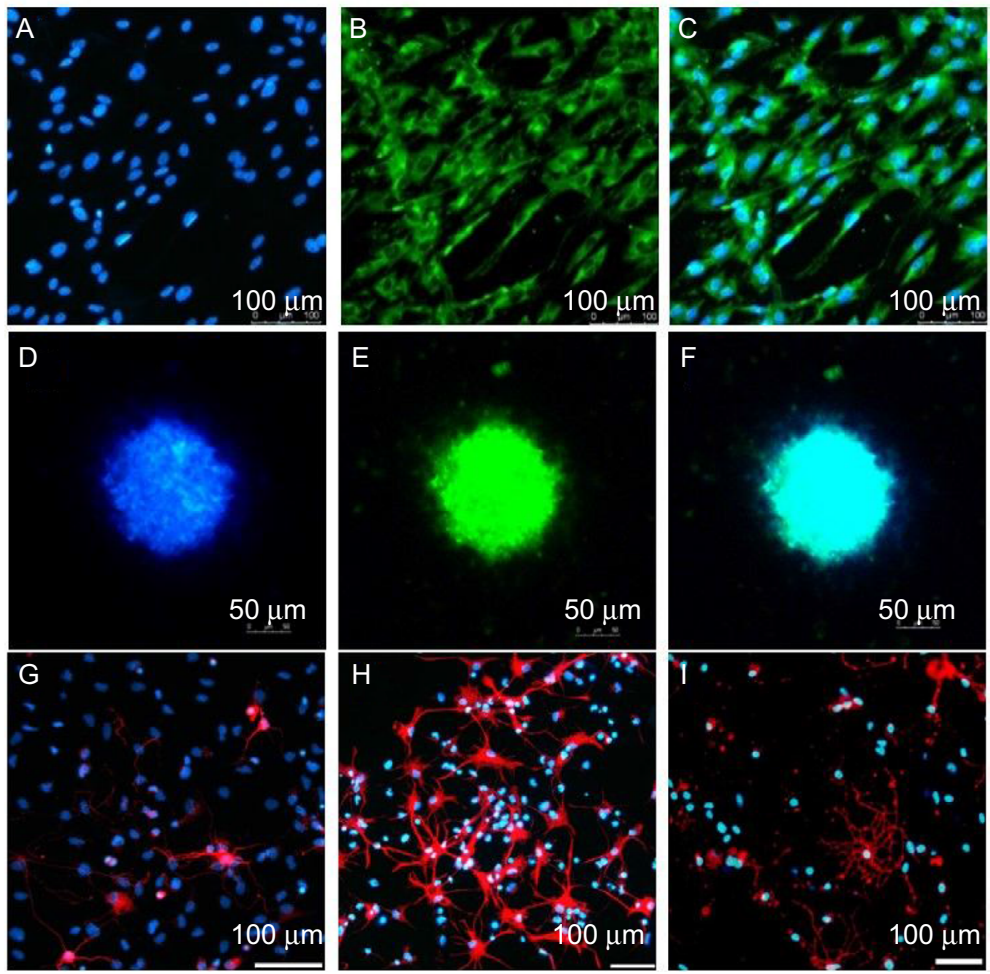

Figure 2 Identity of (A-C) ASCs and (D-F) NSCs. Differentiation of NSCs observed using (G) $\beta$ III-tubulin, (H) GFAP and (I) O4

Notes: The cells were observed under a fluorescence microscope. ASCs were stained with S-100 and DAPI, and NSCs were stained with Nestin and DAPI.

Abbreviations: ASCs, activated Schwann cells; NSCs, neural stem cells.

Figure 2D-F, neurospheres expressed Nestin. NSCs could differentiate into neurons, astrocytes and oligodendrocytes, as examined through $\beta$ III-tubulin, GFAP and $\mathrm{O} 4$ immunofluorescence staining; most of the NSCs differentiated into astrocytes and expressed GFAP, while few expressed $\beta$ III-tubulin or O4. The differentiation rate of neurons was $5.91 \pm 0.94 \%$ (Figure $2 \mathrm{G}-\mathrm{I}$ ).

\section{Best density of ASCs for culturing on PCL scaffold}

In order to determine the suitable density of ASCs for culturing on PCL scaffolds, $2 \times 10^{4}, 8 \times 10^{3}$ and $6 \times 10^{3}$ ASCs were cultured separately on PCL. After 3 days of culture on the scaffolds, crystal violet staining was performed. The results showed that the cells were effectively connected and grew 
well at the density of $2 \times 10^{4} / \mathrm{cm}^{2}$ (Figure $3 \mathrm{~A}-\mathrm{D}$ ), and the number of cells in $2 \times 10^{4} / \mathrm{cm}^{2}$ group was largest compared with the other two groups after 3 days of culture $(P<0.001)$ (Figure 3F), and there was a certain number of ASCs distributed along the longitudinal axis of fibers (Figure $3 \mathrm{E}$ ).

\section{D culture of ASCs on PCL scaffolds}

Confocal microscopy showed that the spindle ASCs could grow well on PCL scaffolds. The cells exhibited spindle shape when examined through S-100/DAPI immunofluorescence staining (Figure 4A-C), which was consistent with the results
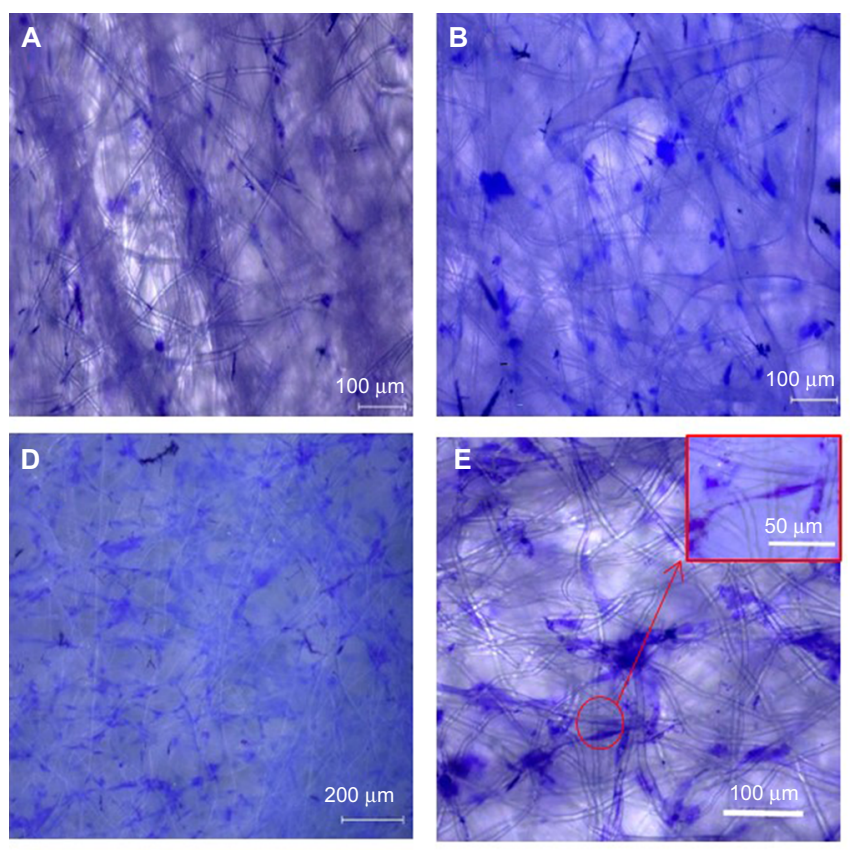

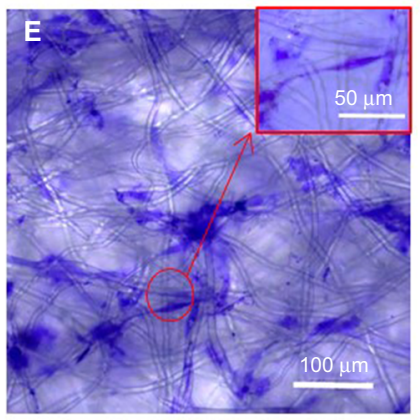

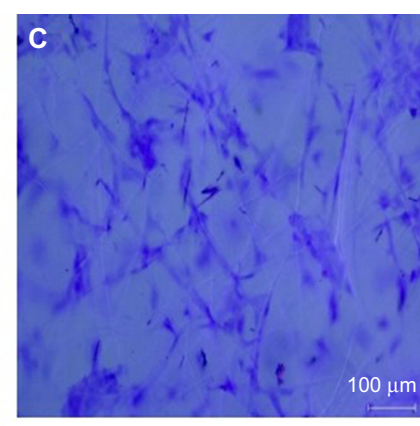

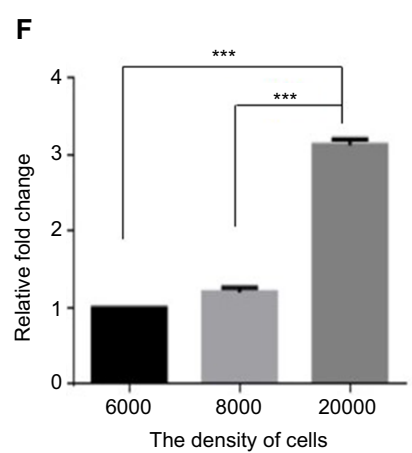

Figure 3 The best density of ASCs cultured in scaffolds: (A) $6 \times 10^{3}$, (B) $8 \times 10^{3}$ and (C-E) $2 \times 10^{4}$.

Notes: Cells were observed by crystal violet staining, and the density of cells was counted by the area of purple by Image-Pro 6 . The ratios between different groups are shown in F. $* * * P<0.001$.

Abbreviation: ASCs, activated Schwann cells.
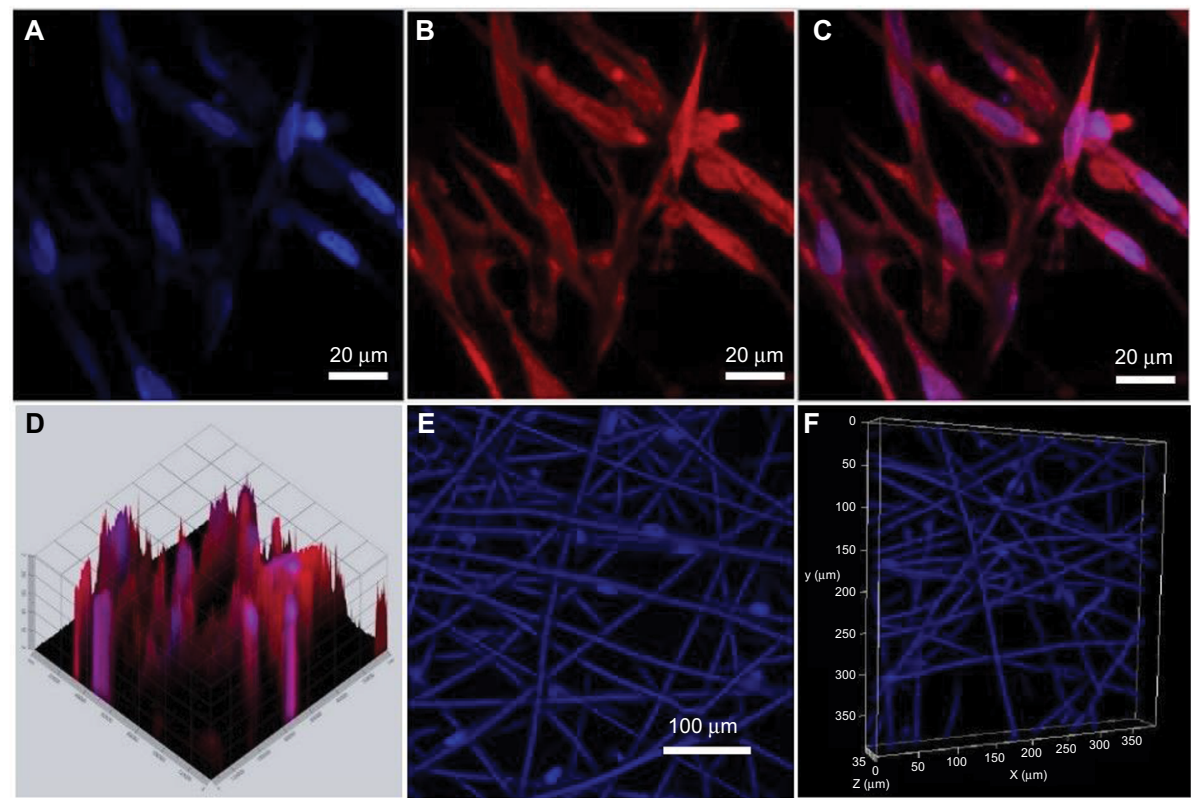

Figure 4 ASCs cultured on PCL fiber scaffold.

Notes: (A-C) S-100 and DAPI staining. (D) The 2.5D picture of ASCs on scaffolds. (E-F) DAPI staining after cells grew on scaffolds for 3 days. The cells were observed under a confocal microscope.

Abbreviations: ASCs, activated Schwann cells; PCL, polycaprolactone. 
of crystal violet staining. The results of DAPI immunofluorescence staining showed that the oval nucleus distributed along the PCL fibers (Figure 4E), and also, as shown in Figure 4D and F, the cells could grow into the inside of the scaffold, and not just on the surface. Cell proliferation was detected by CCK-8 test from 1 to 7 days. The proliferation rate of ASCs was slow from 1st to 3rd day. Nonetheless, the proliferation rate was significantly higher than the first three days from 5 th to 7 th days. The OD reached the peak value on the 7 th day (Figure $5 \mathrm{~A}$ ).

\section{D culture of NSCs on PCL scaffolds}

CCK-8 test was used to detect the proliferation of NSCs on PCL scaffolds. From 1st to 3rd day, the proliferation of NSCs was slow, and the proliferation rate was higher than the previous day, and the growth speed was slow from 5 th to 7 th day. The OD was maximum on the 7th day (Figure 5B).

NSCs were cultured on the scaffolds by using the differentiation medium, and the cells differentiated for 7 days. $\beta$ III-tubulin, GFAP and O4 were detected by immunofluorescence staining. The effects of PCL fiber scaffolds on the ability of NSCs to differentiate into three different cell types were observed by confocal laser scanning microscopy. During 7 days, most of the NSCs differentiated into astrocytes, some into neurons and a few into oligodendrocytes. It was observed that the neurons extended long axons, and the cells were surrounded by neurites. Through double-immunofluorescence staining of $\beta$ III-tubulin and GFAP, we found that most cells were astrocytes, and neurons were surrounded with astrocytes. The differentiation rate of neurons was $24.89 \pm 1.20 \%$

A

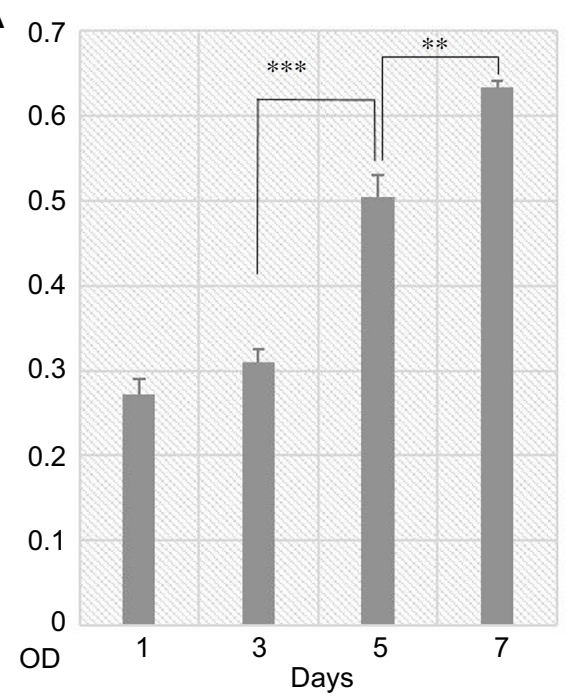

(Figure 6). A variety of cell morphologies were seen through SEM (Figure 7).

\section{Coculture of NSCs and ASCs on PCL scaffolds}

ASCs grew rapidly from the 3rd day when cultured on the scaffolds, which indicated that ASCs adapted to the PCL fiber material at the 3rd day. Thus, ASCs were cultured on PCL scaffold for 3 days first, and then NSCs and ASCs were cocultured on the scaffold for 7 days, and observed by immunofluorescence staining and confocal microscopy. The results showed that ASCs and NSCs were able to grow on PCL. MBP staining showed that ASCs were able to express MBP in 1 week, and NSCs could differentiate into neurons, and the neurons attached to ASCs expressing MBP, indicating that ASCs could express MBP on the PCL material to support axons of neurons (Figure 8). In addition, the coculture system produced more extracellular matrix components on the scaffolds (Figure 9).

\section{Discussion}

In this study, both NSCs and ASCs were found to proliferate well on PCL scaffolds. Neurons derived from NSCs could grow well and distribute around the ASCs, and the axons could extend along with ASCs which expressed MBP. These results suggested the good biocompatibility of PCL scaffold.

ASCs have been successfully applied in the repair of peripheral and central nervous system. However, the low survival rate of the transplanted cells was a major problem. Thus, the repair effect was limited. ${ }^{33-35}$ The loss of the

B

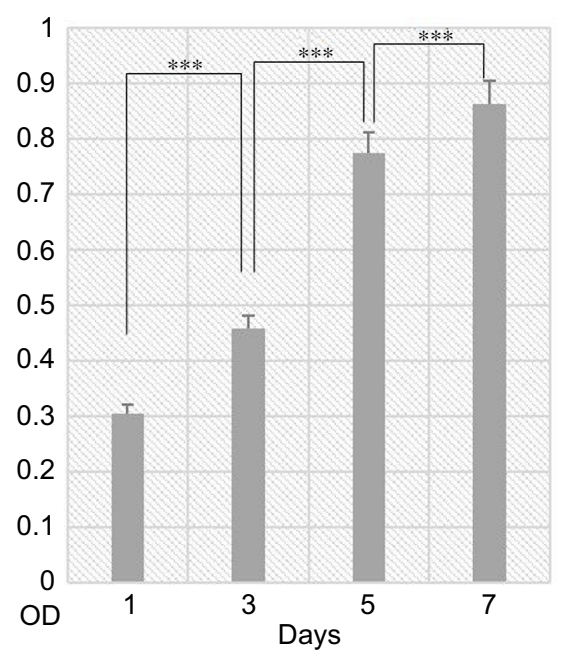

Figure 5 NSCs and ASCs were grown on scaffolds separately.

Notes: CCK-8 test was used to evaluate the proliferation on I, 3, 5 and 7 days: (A) ASCs and (B) NSCs. Mean \pm standard deviation was used in statistical analysis: $* * * P<0.001 ; * * P=0.001$.

Abbreviations: ASCs, activated Schwann cells; CCK-8, cell counting kit-8; NSCs, neural stem cells; OD, optical density. 

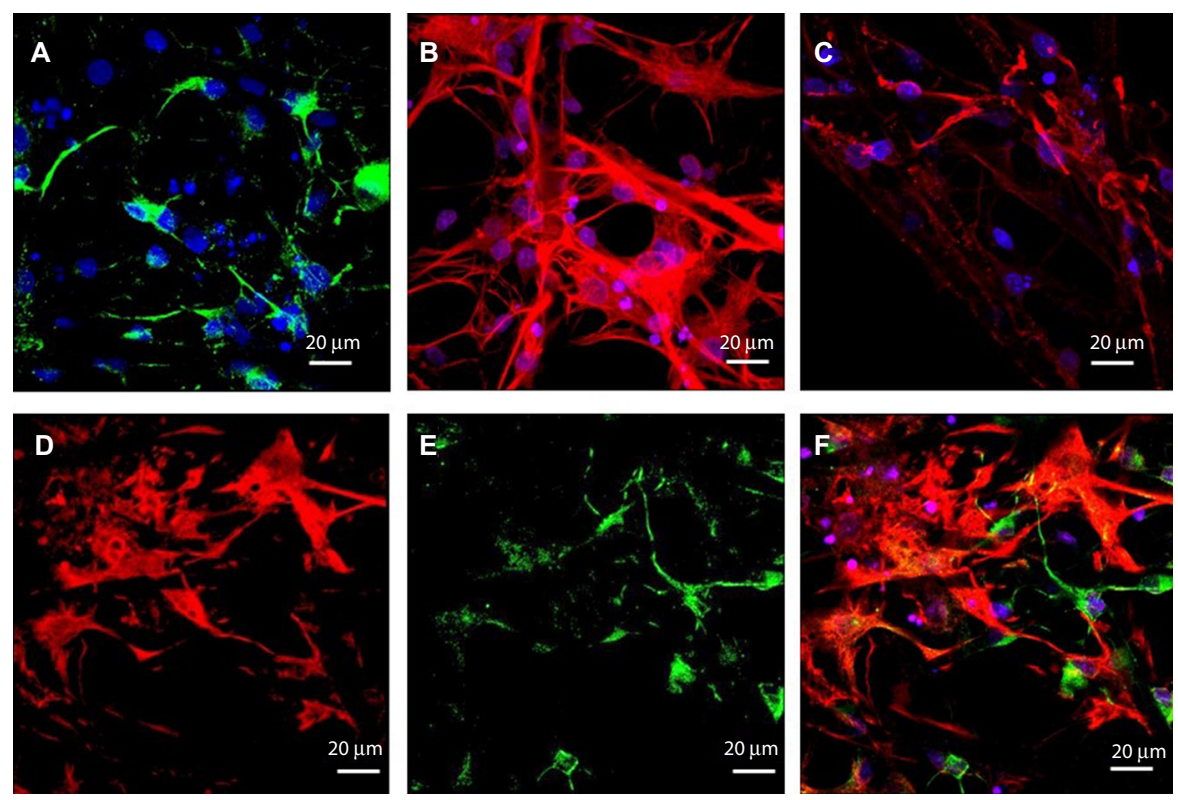

Figure 6 NSCs cultured on PCL fiber scaffolds.

Notes: The cells were observed under a confocal microscope. NSCs differentiated into (A) neurons ( $\beta$ III-tubulin, green), (B) astrocytes (GFAP, red) and (C) oligodendrocytes (O4, red). Staining with (D) GFAP (red) and (E) Blll-tubulin (green). (F) Double staining.

Abbreviations: NSCs, neural stem cells; PCL, polycaprolactone.
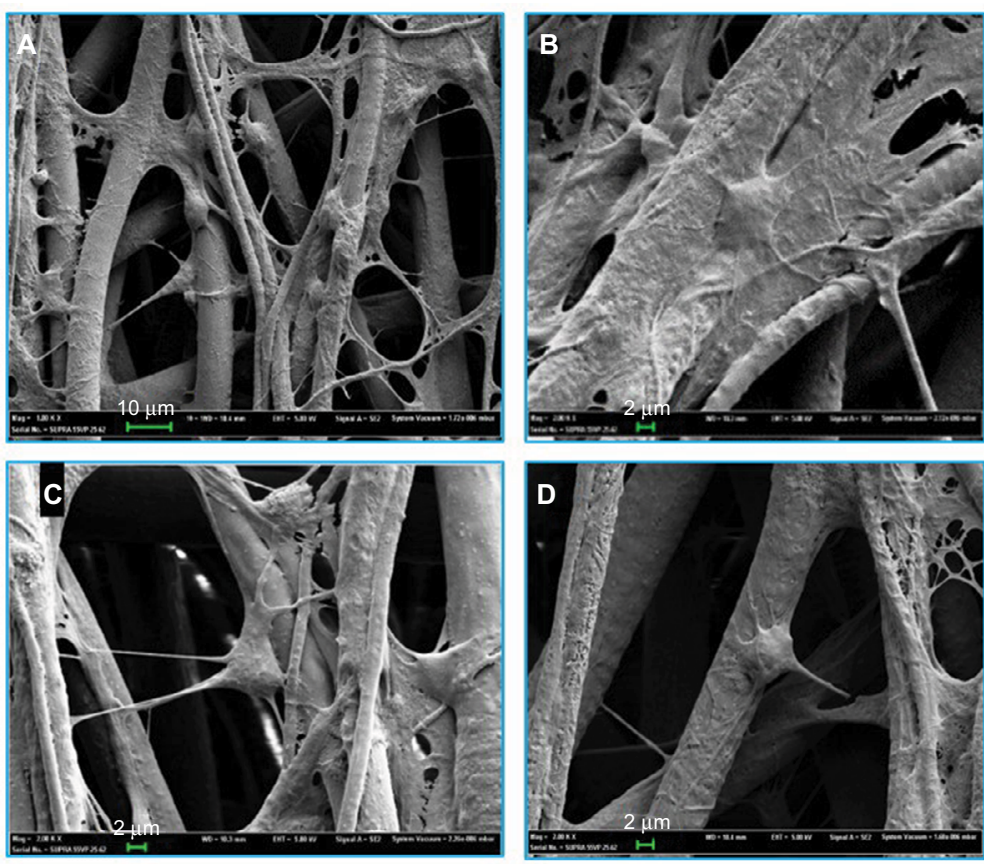

Figure 7 NSCs cultured on scaffolds observed by SEM.

Note: (A) Cells were connected with each other and could grow in the scaffolds. (B) The cell with several neurites and a long neurite. (C) The cell with several of the same neurites, which adhered with adjacent fibers. (D) The long neurite of the cell could extend along with the fiber.

Abbreviations: NSCs, neural stem cells; SEM, scanning electron microscopy.

transplanted cells was mainly due to cell apoptosis, which occurred within the first 3 weeks after transplantation. ${ }^{34}$ The microenvironment after injury was adverse for the survival of transplanted cells, and the presence of low oxygen content and ROS generation and inflammatory cytokines led to cellular death. ${ }^{34}$ The loss of trophic factors as well as the destruction of the matrix for cell adhesion would result in the phenomenon of anoikis, which may also affect the survival of transplanted cells. ${ }^{36}$ The main function of the biomaterial scaffold is supporting the growth of cells, remodeling the anatomical structure and improving functional recovery after nervous system injury. In the present study, randomly distributed fibers simulated the microenvironment in vivo, and our results showed that cells could grow well on the 

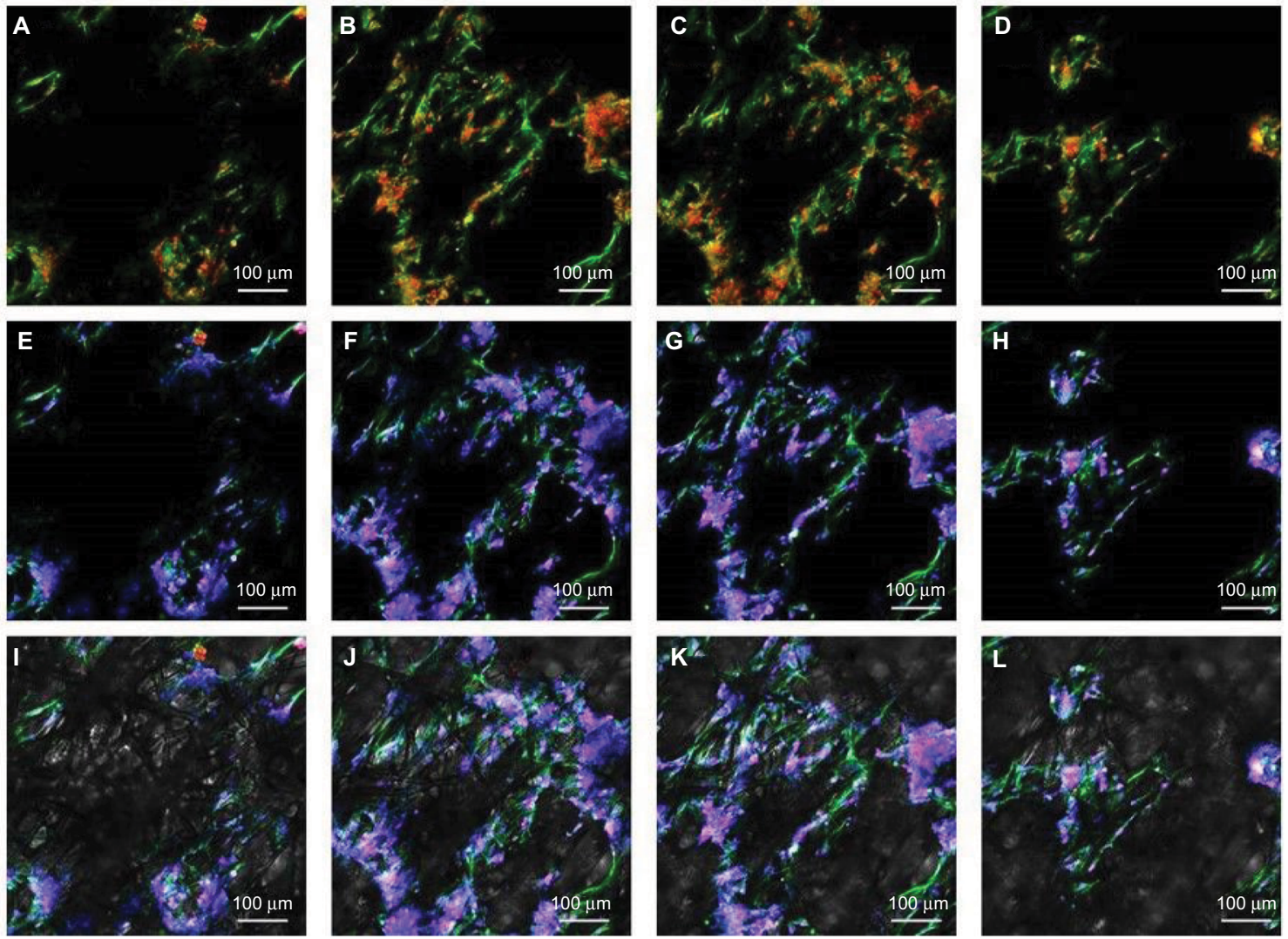

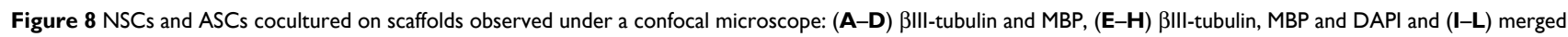
with PCL fiber.

Note: The panels show continuous images of different levels of scaffolds from left to right.

Abbreviations: MBP, myelin basic protein; NSCs, neural stem cells; ASCs, activated Schwann cells; PCL, polycaprolactone.
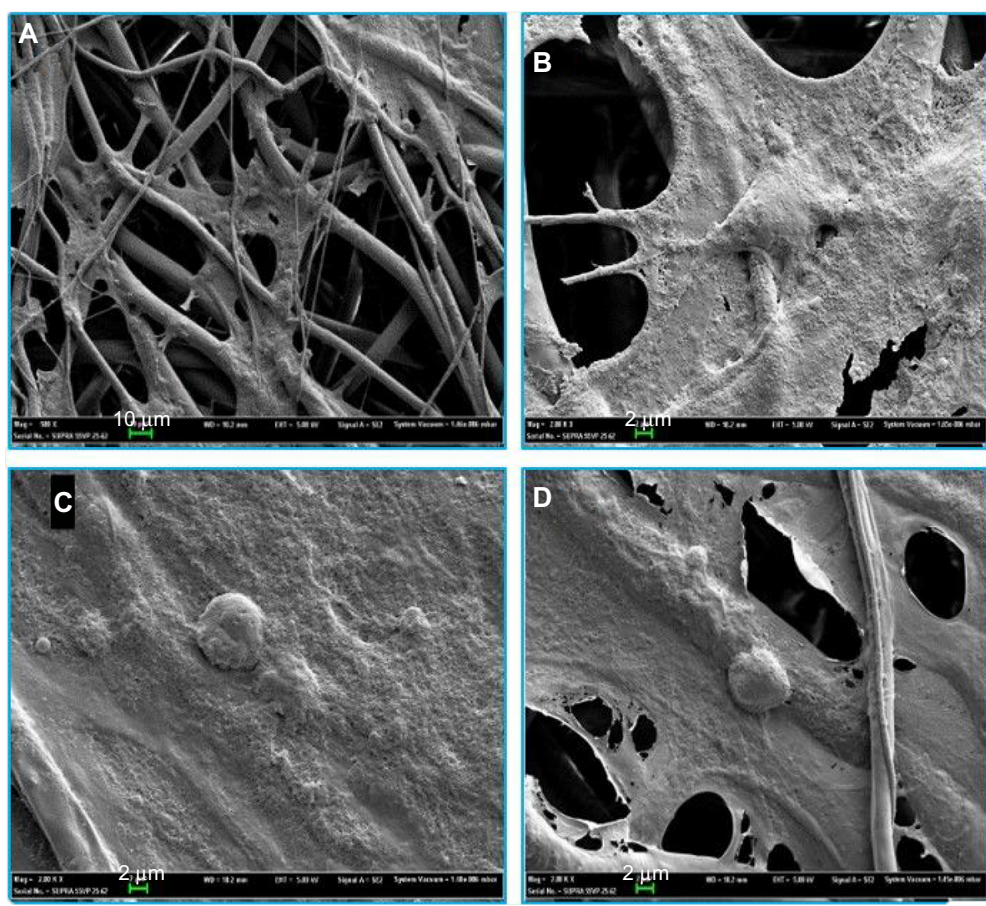

Figure 9 Coculture system on scaffolds observed by SEM.

Notes: (A) Cells adhered in the fibers and the scaffolds could form a 3D culture system. (B) Cells were overlapped with each other. (C) Cells without obvious neurites. (D) Cells could create more extracellular matrix.

Abbreviation: SEM, scanning electron microscopy. 
scaffolds, and the long axis of the cells could extend along the fibers, which indicated the inducing effect of the fibers on cell distribution.

NSCs, as the most promising candidate for repair of the nervous system, have the ability to differentiate into neurons, astrocytes and oligodendrocytes. Several studies have cocultured NSCs and stem cells (SCs) to figure out the effect of Schwann cells on NSCs. Niapour et al cocultured human embryonic SC-derived neural progenitor cells (NPCs) and Schwann cells for 7 days, and found that the rate of expression of GFAP marker was lower, and the rate of expression of TUJ1 and MAP2 marker was higher in the coculture group than the NPCs cultured without SCs. ${ }^{37}$ In addition, some studies demonstrated that SC transplantation could promote the formation of myelin, improve the maturation of regenerated axons and suppress the formation of glia scar and cavity in spinal cord injury. ${ }^{38,39}$ Studies have focused on different methods for coculturing ASCs and NSCs. Thompson and Buettner made an oriented Schwann cell monolayer by micro-patterns made of laminin, and added NSCs in the monolayer. They found that Schwann cells could direct neurite outgrowth, ${ }^{40}$ which proved that the topography and morphology formed by Schwann cells could affect axons of NSCs. ASCs also played an important role in providing support for axons. When NSCs and SCs are cocultured on PLGA material with NT-3 distribution, NSCs more effectively differentiate into neurons, neurons form connections and effective synapses and Schwann cells could express MBP and provide myelin wrapping ${ }^{41}$. This phenomenon was also found in our experiment, in which ASCs provided myelin support for axons. In addition, a study cocultured NSCs with astrocytes. Sørensen et al proved that cells in the central nervous system could survive, differentiate and form myelin in directional PCL grooves and ridges, and when cultured on a layer of astrocytes on PCL, cell survival time and neurite extension length was better than the lysine group and the simple PCL group. ${ }^{42}$ In all of these studies, a layer of cells was prepared, as a feeder, to support the survival and differentiation of SCs. Thus, ASCs were cultured on PCL scaffolds first, and then NSCs were added on the scaffold. Our experiments showed that the proliferation of ASCs accelerated on the 3rd day of culture on the scaffold, which indicated that ASCs adapted to the scaffold; thus, we choose to add NSCs on the 3rd day so that ASCs could provide a good support for NSCs. PCL fiber scaffolds could form a $3 \mathrm{D}$ culture system, indicating that PCL scaffold is a good cell carrier, providing support for the cells. These results lay a foundation for further experiment in vivo.
Generally, the fiber diameter of the electrospinning PCL scaffolds used in other studies was between 400 and 600 $\mathrm{nm} .^{43,44}$ The proliferation and activity of the cells on the scaffold reduced with the increase of fiber diameter. ${ }^{45}$ In this study, we found that ASCs and NSCs could proliferate on the scaffold well, which indicated that cells could survive on this scaffold (fiber diameter, 7.93 $\pm 1.41 \mu \mathrm{m}$ ). The fibers with diameters between 400-500 $\mathrm{nm}$ were easier to adhere; however, the 3D space was not enough. The scaffolds consisting of fibers with diameter in the nanometers range cannot form a 3D culture system effectively. Our present study showed that PCL fibers distributed randomly and formed a 3D culture system, and ASCs could adhere well on the scaffold, and provide a better matrix for NSCs adhesion. Further, the $3 \mathrm{D}$ culture system mimicked the in vivo microenvironment, and there was difference between the SCs cultured in 2D and 3D system. ${ }^{46}$ We also found that after 7 days of coculture, the cells were able to grow into the material. In this study, neuronal axons were able to grow along with Schwann cells, but the fibers of the PCL scaffolds were randomly allocated.

\section{Limitations}

Our study had some shortcomings which need to be solved in the further study. There is still a need to explore the inducing effect of ASCs on the differentiation of NSCs, the inducing effect of lined fibers on the distribution of cells and the repairing effect of coculture system with PCL on nervous system in vivo. However, according to the results of this study, a biodegradable material like PCL could act as a promising scaffold to support the coculture of ASCs and NSCs for nervous system repair.

\section{Conclusion}

Electrospinning PCL scaffolds have a good biocompatibility, and thus, ASCs and NSCs could grow well on these scaffolds, and the ASCs could distribute along the fibers. NSCs cocultured with ASCs on electrospinning PCL scaffolds could form a 3D culture system, and NSCs can differentiate into neurons distributed around ASCs expressing MBP. Thus, PCL scaffolds can be used as a cell carrier for in vivo transplantation.

\section{Acknowledgments}

This study was supported by the State Key Program of National Natural Science Foundation of China (81330042), Special Program for Sino-Russian Joint Research Sponsored by the Ministry of Science and Technology, China (2014DFR31210), International Cooperation Program of National Natural Science Foundation of China (81620108018), and Key 
Program Sponsored by the Tianjin Science and Technology Committee, China (14ZCZDSY00044, 13RCGFSY19000). The authors also thank Yan Hao and Baowen Liu for their help with the experiment.

\section{Disclosure}

The authors report no potential conflicts of interest in regard to the research, authorship and/or publication of this work.

\section{References}

1. Mehrabi S, Eftekhari S, Moradi F, et al. Cell therapy in spinal cord injury: a mini-review. Basic Clin Neurosci. 2013;4(2):172-176.

2. Barnabé-Heider F, Frisén J. Stem cells for spinal cord repair. Cell Stem Cell. 2008;3(1):16-24.

3. Tremp M, Meyer Zu Schwabedissen M, Kappos EA, et al. The regeneration potential after human and autologous stem cell transplantation in a rat sciatic nerve injury model can be monitored by MRI. Cell Transplant. 2015;24(2):203-211.

4. Wei L, Wei ZZ, Jiang MQ, Mohamad O, Yu SP. Stem cell transplantation therapy for multifaceted therapeutic benefits after stroke. Prog Neurobiol. Epub 2017 Mar 18.

5. Ronaghi M, Erceg S, Moreno-Manzano V, Stojkovic M. Challenges of stem cell therapy for spinal cord injury: human embryonic stem cells, endogenous neural stem cells, or induced pluripotent stem cells? Stem Cells. 2010;28(1):93-99.

6. Wiliams RR, Bunge MB. Schwann cell transplantation: a repair strategy for spinal cord injury? Prog Brain Res. 2012;201:295-312.

7. Silva NA, Sousa N, Reis RL, Salgado AJ. From basics to clinical: a comprehensive review on spinal cord injury. Prog Neurobiol. 2014;114:25-57.

8. Zhang Q, Nguyen P, Xu Q, et al. Neural progenitor-like cells induced from human gingiva-derived mesenchymal stem cells regulate myelination of Schwann cells in rat sciatic nerve regeneration. Stem Cells Transl Med. 2017;6(2):458-470.

9. Barnett SC, Riddell JS. Olfactory ensheathing cells (OECs) and the treatment of CNS injury: advantages and possible caveats. J Anat. 2004;204(1):57-67.

10. Liang P, Liu J, Xiong J, et al. Neural stem cell-conditioned medium protects neurons and promotes propriospinal neurons relay neural circuit reconnection after spinal cord injury. Cell Transplant. 2014;23 (Suppl 1):S45-S56.

11. Lu P, Wang Y, Graham L, et al. Long-distance growth and connectivity of neural stem cells after severe spinal cord injury. Cell. 2012;150(6):1264-1273.

12. Sabelström H, Stenudd M, Réu P, et al. Resident neural stem cells restrict tissue damage and neuronal loss after spinal cord injury in mice. Science. 2013;342(6158):637-640.

13. Tao Li J, Somasundaram C, Bian K, et al. Nitric oxide signaling and neural stem cell differentiation in peripheral nerve regeneration. Eplasty. 2010;10:e42.

14. Dadon-Nachum M, Melamed E, Offen D. Stem cells treatment for sciatic nerve injury. Expert Opin Biol Ther. 2011;11(12):1591-1597.

15. Piltti KM, Avakian SN, Funes GM, et al. Transplantation dose alters the dynamics of human neural stem cell engraftment, proliferation and migration after spinal cord injury. Stem Cell Res. 2015;15(2):341-353.

16. Song S, Kamath S, Mosquera D, et al. Expression of brain natriuretic peptide by human bone marrow stromal cells. Exp Neurol. 2004;185(1):191-197.

17. Wu S, Suzuki Y, Kitada M, et al. Migration, integration, and differentiation of hippocampus-derived neurosphere cells after transplantation into injured rat spinal cord. Neurosci Lett. 2001;312(3):173-176.

18. Houweling DA, Lankhorst AJ, Gispen WH, Bär PR, Joosten EA. Collagen containing neurotrophin-3 (NT-3) attracts regrowing injured corticospinal axons in the adult rat spinal cord and promotes partial functional recovery. Exp Neurol. 1998;153(1):49-59.
19. Enomoto M. Therapeutic effects of neurotrophic factors in experimental spinal cord injury models. J Neurorestoratol. 2016;4(1):15-22.

20. Fon D, Zhou K, Ercole F, et al. Nanofibrous scaffolds releasing a small molecule BDNF-mimetic for the re-direction of endogenous neuroblast migration in the brain. Biomaterials. 2014;35(9):2692-2712.

21. Akassoglou K, Yu WM, Akpinar P, Strickland S. Fibrin inhibits peripheral nerve remyelination by regulating Schwann cell differentiation. Neuron. 2002;33(6):861-875.

22. Keilhoff G, Fansa H, Schneider W, Wolf G. In vivo predegeneration of peripheral nerves: an effective technique to obtain activated Schwann cells for nerve conduits. J Neurosci Methods. 1999;89(1):17-24.

23. Pearse DD, Barakat DJ. Cellular repair strategies for spinal cord injury. Expert Opin Biol Ther. 2006;6(7):639-652.

24. Wang JM, Zeng YS, Wu JL, Li Y, Teng YD. Cograft of neural stem cells and schwann cells overexpressing TrkC and neurotrophin-3 respectively after rat spinal cord transection. Biomaterials. 2011;32(30):7454-7468.

25. Yang Y, Kamudzandu M, Roach P, Fricker R. Nanofibrous scaffolds supporting optimal central nervous system regeneration: an evidencebased review. J Neurorestoratol. 2015;3:123-131.

26. Middleton JC, Tipton AJ. Synthetic biodegradable polymers as orthopedic devices. Biomaterials. 2000;21(23):2335-2346.

27. Ng KW, Achuth HN, Moochhala S, Lim TC, Hutmacher DW. In vivo evaluation of an ultra-thin polycaprolactone film as a wound dressing. J Biomater Sci Polym Ed. 2007;18(7):925-938.

28. Woodruff MA, Hutmacher DW. The return of a forgotten polymerpolycaprolactone in the 21st century. Prog Polym Sci. 2010;35(10): 1217-1256.

29. Kim DY, Choi YS, Kim SE, et al. In vivo effects of adipose-derived stem cells in inducing neuronal regeneration in Sprague-Dawley rats undergoing nerve defect bridged with polycaprolactone nanotubes. J Korean Med Sci. 2014;29 (Suppl 3):S183-S192.

30. Donoghue PS, Lamond R, Boomkamp SD, et al. The development of a $\varepsilon$-polycaprolactone scaffold for central nervous system repair. Tissue Eng Part A. 2013;19(3-4):497-507.

31. Beigi MH, Ghasemi-Mobarakeh L, Prabhakaran MP, et al. In vivo integration of poly( $\varepsilon$-caprolactone)/gelatin nanofibrous nerve guide seeded with teeth derived stem cells for peripheral nerve regeneration. J Biomed Mater Res A. 2014;102(12):4554-4567.

32. Azari H, Sharififar S, Rahman M, Ansari S, Reynolds BA. Establishing embryonic mouse neural stem cell culture using the neurosphere assay. JVis Exp. 2011;(47):pii 2457.

33. Cho K-S, Pearse DD, Deitrich DW, et al. Schwann cell transplantation improves reticulo-spinal fiber growth and forelimb strength after severe cervical spinal cord contusion. Exp Neurol. 2007;193:239.

34. Hill CE, Hurtado A, Blits B, et al. Early necrosis and apoptosis of Schwann cells transplanted into the injured rat spinal cord. Eur J Neurosci. 2007;26(6):1433-1445.

35. Barakat DJ, Gaglani SM, Neravetla SR, et al. Survival, integration, and axon growth support of glia transplanted into the chronically contused spinal cord. Cell Transplant. 2005;14(4):225-240.

36. Pearse DD, Sanchez AR, Pereira FC, et al. Transplantation of Schwann cells and/or olfactory ensheathing glia into the contused spinal cord: survival, migration, axon association, and functional recovery. Glia. 2007;55(9):976-1000.

37. Niapour A, Karamali F, Nemati S, et al. Cotransplantation of human embryonic stem cell-derived neural progenitors and schwann cells in a rat spinal cord contusion injury model elicits a distinct neurogenesis and functional recovery. Cell Transplant. 2012;21(5):827-843.

38. Wang X, Xu XM. Long-term survival, axonal growth-promotion, and myelination of Schwann cells grafted into contused spinal cord in adult rats. Exp Neurol. 2014;261:308-319.

39. Bunge MB. Efficacy of Schwann cell transplantation for spinal cord repair is improved with combinatorial strategies. J Physiol. 2016;594(13):3533-3538.

40. Thompson DM, Buettner HM. Neurite outgrowth is directed by schwann cell alignment in the absence of other guidance cues. Ann Biomed Eng. 2006;34(1):161-168. 
41. Xiang L, Chen Y. Stem cell transplantation for treating spinal cord injury: a literature comparison between studies of stem cells obtained from various sources. Neural Regen Res. 2012;7(16):1256-1263.

42. Sørensen A, Alekseeva T, Katechia K, Robertson M, Riehle MO, Barnett SC. Long-term neurite orientation on astrocyte monolayers aligned by microtopography. Biomaterials. 2007;28(36):5498-5508.

43. Ebrahimi-Barough S, Hoveizi E, Yazdankhah M, et al. Inhibitor of $\mathrm{PI} 3 \mathrm{~K} /$ Akt signaling pathway small molecule promotes motor neuron differentiation of human endometrial stem cells cultured on electrospun biocomposite polycaprolactone/collagen scaffolds. Mol Neurobiol. 2016;54(4):2547-2554.
44. Raspa A, Marchini A, Pugliese R, et al. A biocompatibility study of new nanofibrous scaffolds for nervous system regeneration. Nanoscale. 2016;8(1):253-265.

45. Wang J, Ye R, Wei Y, et al. The effects of electrospun TSF nanofiber diameter and alignment on neuronal differentiation of human embryonic stem cells. J Biomed Mater Res A. 2012;100(3):632-645.

46. Kamei KI, Koyama Y, Tokunaga Y, et al. Characterization of phenotypic and transcriptional differences in human pluripotent stem cells under 2D and 3D culture conditions. Adv Healthc Mater. 2016;5(22): 2951-2958.
Journal of Neurorestoratology

\section{Publish your work in this journal}

The Journal of Neurorestoratology is an international, peer-reviewed, open access online journal publishing original research and review articles on the subject of Neurorestoratology. To provide complete coverage of this revolutionary field the Journal of Neurorestoratology will report on relevant experimental research, technological advances,

\section{Dovepress}

and clinical achievements. The manuscript management system is completely online and includes a very quick and fair peer-review system, which is all easy to use. Visit http://www.dovepress.com/testimonials. php to read real quotes from published authors. 